\title{
The Quality Analysis and Exploration of the Examination Papers of Occupational Safety and Health from the Perspective of Empirical Analysis
}

\author{
Dongping Shi ${ }^{\mathrm{a}}$, Chengyu $\mathrm{Xie}^{\mathrm{b}}$ and $\mathrm{Hao} \mathrm{Lu}{ }^{\mathrm{c}}$ \\ College of Environment and Resources, Xiangtan University, Xiangtan 411105, China \\ a2029931597@qq.com, bxiechengyu42@163.com, c187194616@qq.com
}

\begin{abstract}
The quality analysis of safety engineering major "Occupational Safety and Health" paper is carried out, which provides the basis for further improving teaching quality and teaching reform. EXCEL and SPSS software were used to analyze 50 test paper samples of safety engineering specialty. The contents of the analysis included: score distribution statistics, test paper structure and value distribution, difficulty degree and differentiation degree. The analysis results show that the test results basically accord with the normal distribution, the objective examination questions of the course are moderate in difficulty and the degree of distinction is good, which can reflect that the students' mastery of the course meets the requirements of the non-selective examination.
\end{abstract}

Keywords: Examination paper analysis; Quantitative analysis; Teaching evaluation; Occupational safety and health.

\section{Introduction}

Improving teaching quality is the eternal theme of educational reform in colleges and universities. As an important part of course teaching evaluation, final examination plays an important role in teaching effect evaluation. The analysis of test paper quality is that teachers use mathematical statistics to analyze the test paper, dig out the effective information in the test paper, help the teacher to grasp the studentsundefined effect quickly, and improve the teaching quality of the course.

Occupational safety and health is the basic theory course of safety engineering. Through the course study, students can master the basic concept, hazard identification, protection method and control means of occupational hazards in industrial production. In order to promote the teaching quality of safety engineering major in our university, the final examination paper of Occupational Safety and Health was selected as the research object to analyze the quality of the test paper. Through the analysis of test paper quality, it can provide the evaluation basis for the teaching activity and proposition work of the course, optimize the teaching decision, and promote the benign development of the teaching reform.

\section{Research Objects and Methods}

\subsection{Research Objects}

The subjects of this study were 50 students majoring in safety engineering in a certain grade. The teaching materials were selected as the 11th Five-Year Plan textbook of the safety engineering series of higher education-Occupational Health and Protection (edited by Mechanical Industry Press, Chen Yuanjiang, Wu Chao, Wu Guixiang). The examination method is closed examination, test paper score is 100 , examination time is 120 minutes. According to the outline requirements, the final examination papers are assigned according to the teaching time, and the teachers make the proposition according to the test outline requirements. Test papers are judged in strict accordance with the answers and scoring criteria established before the examination. Fifty final examination papers of Occupational Safety and Health were selected as samples for analysis. 


\subsection{Research Methods}

SPSS and EXCEL were used to analyze the data of 50 samples. The paper mainly analyzes the examination paper grade distribution, examination paper difficulty and the distinguish degree and so on content.

Distribution of total scores:The highest and lowest score, the average score, the pass rate and the overall distribution of the test paper.

Difficulty of examination paper:The difficulty is a reflection of the degree of difficulty and ease of the test, and the difficulty coefficient $\mathrm{P}$ denotes, $\mathrm{P}<0.7$ is more difficult problem, $0.7 \leq \mathrm{P}<0.8$ is a moderate problem. $8 \leq \mathrm{P}<1$ is an easy problem.

Classification of examination papers:The degree of distinction is the degree to which the examine student actual level is distinguished, that is to say, it can draw apart the level of student performance. $\mathrm{D} \geq 0.4$ for excellent examination questions. $0.3 \leq \mathrm{D}<0.4$ for good examination questions, $0.2 \leq \mathrm{D}$ $<0.3$ for general examination questions , $\mathrm{D}<0.2$ for bad examination questions.

\section{Research Content and Results}

\subsection{Score Distribution}

The number of candidates for the examination is 50 and the actual number is 50 . The results are distributed in Table 1, the average score is 72.0, the average score is between 51 and 95, excellent 1 , good 13, medium 22, passing 8, failing 3 .

Table 1. Distribution of examination results

\begin{tabular}{ccccc}
\hline Score distribution & Number of students & Proportion & Average score & Passing rate \\
\hline $90-100$ (excellent) & 1 & $2.0 \%$ & & \\
$80-89$ (good) & 13 & $26.5 \%$ & & \\
$70-79$ (medium) & 22 & $44.9 \%$ & 72.0 & $89.8 \%$ \\
60-69(passing) & 8 & $16.3 \%$ & & \\
Under 59(failing) & 3 & $6.1 \%$ & & \\
\hline
\end{tabular}

\subsection{Examination Paper Structure and Score Distribution}

The types of test papers include multiple-choice questions, fill-in-the-blank questions, noun explanations, short-answer questions, calculation questions, and essay questions. The test scores and questions are shown in Table 2.

Table 2. Exam questions and score distribution list

\begin{tabular}{lcccc}
\hline \multicolumn{2}{c}{ question types } & The topic quantity & score & Score ratio(\%) \\
\hline \multirow{3}{*}{ Objective questions } & multiple-choice questions & 10 & 20 & 20 \\
& fill-in-the-blank questions & 10 & 10 & 10 \\
& noun explanations & 4 & 16 & 16 \\
\cline { 2 - 5 } Subjective questions & short-answer questions & 4 & 24 & 24 \\
& calculation questions & 1 & 15 & 15 \\
& essay questions & 1 & 15 & 15 \\
\hline
\end{tabular}

The test paper is assigned by the teachers in the research group according to the teaching outline and the teaching plan. The distribution of chapter scores is shown in table 3. 
Table 3. Examination chapter distribution list

\begin{tabular}{ccccc}
\hline Chapter & $\begin{array}{c}\text { Number of } \\
\text { questions }\end{array}$ & $\begin{array}{c}\text { Proportion of } \\
\text { questions(\%) }\end{array}$ & Score & $\begin{array}{c}\text { Proportion of } \\
\text { score }(\%)\end{array}$ \\
\hline Chapter One & 1 & 3.3 & 2 & 2 \\
Chapter Two & 3 & 10 & 10 & 10 \\
Chapter Three & 5 & 16.7 & 10 & 10 \\
Chapter Four & 4 & 13.3 & 15 & 15 \\
Chapter Five & 5 & 16.7 & 18 & 18 \\
Chapter Six & 5 & 16.7 & 19 & 19 \\
Chapter Seven & 2 & 6.7 & 8 & 8 \\
Chapter Eight & 2 & 6.7 & 8 & 8 \\
Chapter Nine & 1 & 3.3 & 6 & 6 \\
Chapter Ten & 1 & 3.3 & 2 & 2 \\
Chapter & 1 & 3.3 & 2 & 2 \\
Eleven & 1 & & & \\
\hline
\end{tabular}

\subsection{Quality Analysis of Test Papers}

Table 4. Difficulty distribution of examination questions

\begin{tabular}{cccc}
\hline Classification & $<0.7$ & $0.7 \sim 0.8$ & $>0.8$ \\
\hline Evaluation & difficult & moderate & easy \\
multiple-choice questions & 0 & 3 & 7 \\
fill-in-the-blank questions & 1 & 3 & 6 \\
Noun explanations & 0 & 0 & 4 \\
short-answer questions & 1 & 2 & 1 \\
calculation questions & 1 & 0 & 0 \\
essay questions & 1 & 0 & 0 \\
total & 4 & 8 & 18 \\
Proportion of questions(\%) & 13.3 & 26.7 & 60 \\
Score & 37 & 21 & 42 \\
\hline
\end{tabular}

Table 5. Test questions discrimination degree distribution table

\begin{tabular}{ccccc}
\hline Classification & $>0.4$ & $0.30 \sim 0.40$ & $0.20 \sim 0.30$ & $<0.2$ \\
\hline Evaluation & excellent & good & general & bad \\
multiple-choice questions & 1 & 2 & 4 & 3 \\
fill-in-the-blank questions & 2 & 2 & 4 & 2 \\
Noun explanations & 0 & 1 & 2 & 1 \\
short-answer questions & 1 & 0 & 2 & 1 \\
calculation questions & 1 & 0 & 0 & 0 \\
essay questions & 1 & 0 & 0 & 0 \\
total & 6 & 5 & 12 & 7 \\
Proportion of questions $(\%)$ & 20 & 16.7 & 40 & 23.3 \\
Score & 39 & 10 & 32 & 19 \\
\hline
\end{tabular}

\section{Whole Analysis of Test Paper}

\subsection{Quality Analysis of Test Papers}

The distribution curve of students' scores is similar to that of normal distribution, with an average score of 72.0 points. Most of the students' scores were distributed in 70 or 79 points, the proportion was 44.9, in which the number of excellent (90-100) was 1 , good (80-89) was 13, medium (70-79) was 22 , passing (60-69) was 8 , and failing (below 59) was 3 . The results of test paper accord with 
normal distribution, which indicates that the students have a good mastery of the course and meet the requirements of non-selective examination

The objective questions of the course are moderate in difficulty and well differentiated. The difficult questions in the examination paper account for $13.3 \%$, with a score of 37 . Moderate topics account for $26.7 \%$, with a score of 21 ; Easy problem accounts for 60 percent, with a score of 42 . Good discrimination accounts for $20 \%$ of the questions, with a score of 39 . Good subjects accounted for $16.7 \%$, with a score of 10 ; The general problem accounts for $40 \%$, with a score of $32 \%$. The difference problem was $23.3 \%$, with a score of 19 ., which can reflect that students' mastery level of this course meets the requirements of non-selective examination.

\subsection{Implications for Test Propositions}

In the course of examination paper proposition, the construction of examination paper database should be constantly improved, and the questions with poor discrimination should be eliminated, so as to enhance the distinction and credibility of the questions. The propositional process should be carried out in combination with the training objectives and teaching contents of the safety engineering major, and with the teaching syllabus and examination syllabus, the suitable question type should be selected for the propositional. The curriculum of occupational safety and health is closely related to practice. It is necessary to increase students' comprehensive application ability in the direction of proposition.

\subsection{Implications for Test Methods}

The traditional examination method is closed-book examination, which lacks the assessment of students' independent learning ability and independent thinking ability. In the examination method, we should pay attention to the examination of students' practical ability. The contents of class participation, class testing, group discussion, comprehensive design and flipping class are combined with the final examination to increase the exploration and construction of the diversified examination.

\section{Conclusion}

The final exam can not only be used to evaluate students' learning, but also evaluate teachers' teaching quality. Through the quality analysis of the final exam paper, it can have a systematic and comprehensive understanding of students' scores, help teachers to master the feedback information of students, timely find the weak links in the teaching process, adjust the teaching content and improve the teaching methods. It provides beneficial reference for carrying out teaching reform and improving teaching quality.

Occupational safety and health, as a basic course of safety engineering, plays an important role in the training of safety engineering professionals. How to carry out teaching reasonably and efficiently is an urgent problem to be solved. Teachers should explore more effective and systematic teaching methods and examination methods according to the characteristics of the course. Through the examination paper analysis of occupational safety and health, new ideas can be provided for the teaching and examination of occupational safety and health.

\section{Summary}

This work is supported by the Teaching Reform and Research Project of Xiangtan University (Reform and Innovation of the Teaching Mode of College students' Public Safety Education course),Hunan Province Teaching Research Reform General Project (Exploration on the Reform of Safety Engineering Education Based on Cloud Information and Data in the Background of New Engineering), Hunan Province Teaching Research Reform General Project (Research on Undergraduate Talents Training Program of Safety Engineering Based on Multidisciplinary Intersection). 


\section{References}

[1]. Cui G S, Zhang N. The major indexes of examination paper analysis and evaluation system as well as some approaches to the key issues[J]. Journal of Shenyang Institute of Engineering, 2011.

[2]. Swart A J. Evaluation of Final Examination Papers in Engineering: A Case Study Using Bloom's Taxonomy[J]. IEEE Transactions on Education, 2010, 53(2):257-264.

[3]. Fabian C L, Teubl S, Binder C R. Evaluation of models for dermal exposure assessment in farming systems in developing countries[J]. Journal of Environmental Engineering \& Ecological Science, 2014, 3(1):1.

[4]. Wang Z Z. Reasons for Choosing Librarianship_-Analysis of Undergraduates' Answers on Final Examination Papers of the Course An Introduction to Library Science[J]. Library Tribune, 2015. 